\title{
Orthopaedic patients' perceptions about their pre-operative information
}

\author{
C Chetty, M Cur (Unisa) \\ Lecturer, King Edward viii Nursing College and M Cur graduate, Department of Health Studies, University of South \\ Africa
}

VJ Ehlers, D Litt et Phil (Unisa)

Professor, Department of Health Studies, University of South Africa

\section{Kev words}

patients' perceptions, pre-operative information, orthopaedic patients

\section{Correspondence address}

Prof VJ Ehlers,

POBox 65075,

ERASMUSRAND. 0165.

Tel/fax:012-347-8287.

E-mail: ehlervj@unisa.ac.za or ehlersjh@mweb.co.za

\section{Abstract: Curationis 32 (4): 55-60}

A non-experimental, descriptive and quantitative survey was conducted to explore orthopaedic patients' perceptions about the pre-operative information received when undergoing elective surgery in two hospitals in the KwaZulu-Natal Province. The findings indicate that most patients perceived the pre-operative information to be useful in their preparation for surgery. Aspects that were not addressed during preoperative information sessions included post-operative nutrition, pain medication, ambulation, deep breathing and coughing exercises. The recommendations include that all these aspects should be addressed in future pre-operative education sessions. Further research should be conducted for enhancing the pre-operative information provided to patients scheduled to undergo elective orthopaedic surgery.

\section{Introduction and background information}

Patients undergoing elective surgery experience fear and anxiety because they do not know what to expect. Preoperative information assists the patient to become more knowledgeable about the prospective surgery and enables the nurse to learn about the patient and to establish rapport before the patient is taken to the operating theatre (Phillips, 2004:368). Pre-operative teaching reduces the patient's apprehension and fear, increases the patient's co-operation and participation in post-operative care and decreases the incidence and severity of postoperative complications (Ignatavicius, Workman \& Mishler, 1999:321-322; Lee \& Chien, 2002:1). Pre-operative information should provide patients with clear instructions preparing them for their surgical procedures and post-operative care. Prouty, Cooper, Thomas, Christensen, Strong, Bowie and
Oermann (2006:257) describe a pre-operative information programme offered to patients undergoing orthopaedic surgery at a hospital in Michigan in the United States of America. The programme covered pre-operative preparation and included aspects such as preparation for surgery and medications, a review of the anatomy of the knee or hip, medical devices patients will encounter in the hospital such as drains and catheters and information on how to prevent complications. Patients reported that this pre-operative teaching was effective in reducing their anxiety levels about their surgery.

According to Lewis, Heitkemper and Dirksen (2004:369), pre-operative teaching encompasses three types of information: sensory, process and procedural. Sensory information relates to what patients see, hear, smell and feel. Process information concerns the general flow of events. For example, the patient may be told that he will be taken 
to the waiting area first, then to the operating room and after surgery to the recovery room before returning to the ward or to the intensive or high care unit, depending on the surgery. Procedural information includes post-operative fluid and food restrictions, expected drains, tubes, catheters and intravenous infusions.

Fitzpatrick and Hyde (2005:254-255) reported that surgical nurses in Ireland, used words to convey pre-operative information rather than teaching tools or aids. However, these Irish nurses reportedly recognised the potential benefits of using visual materials. $\mathrm{Pa}$ tient information leaflets were available but were specific to the post-operative period. Some nurses used diagrams of bones to illustrate an operative procedure such as the insertion of pins.

Johansson, Nuutila, Virtanen and Salantera (2005:220), maintain that booklets, used in pre-operative patient information, enable patients to return to them for information. However, written information alone proved inadequate to provide effective pre-operative information. When conducting preoperative teaching, the nurse should explain the operation, the immediate post-operative period's expectations and the mobilisation plan. Evaluating how well the patient understands the procedure is important. This could be done by asking the patient to explain in his/her own words what the operation entails in writing on the form where he/she signs consent for the surgery.

According to Altizer (2004:283), an organised pre-operative teaching programme should begin with normal anatomy and an explanation of how joints work. Anatomic models showing a normal hip or knee should help the patient to understand the normal anatomy. The patient's own X-rays could be used to show the involved joint with narrowing or complete loss of joint space, fractures and/or displacements. The patient should also be shown a post-operative film of a joint implant in place - if the patient is scheduled for implant surgery. Showing the actual prostheses could also be helpful, but keeping examples for this purpose might be too expensive to be feasible.

Pre-operative teaching improves post- operative compliance (Pellini, Tluczek, Collins, Trimborn, Norwich, Engelke \& Broad, 1998:48; Walker, 2007:27; Whyte \& Grant, 2005:201). Pre-operative teaching also involves discussions with the patient and family about the need for assistance with activities of daily living and the need for support once the patient is discharged. This may involve informing the patient and family on ways to modify the home environment in order to accommodate the altered mobility of the patient after surgery as well as referral to the social worker (Smeltzer \& Bare, 2004: 2041).

Spalding (2004:152) states that pre-operative information has the potential to increase patients' confidence and enable them to take control of their health and well-being. Comprehensive preoperative information should help both the family and the patient understand the procedure and what to expect postoperatively. Pre-operative information also assists the patient to set realistic recuperation goals.

\section{Problem statement}

Pre-operative information was not provided regularly by nurses in the two hospitals where this research was conducted. The first author, a nurse educator teaching operating theatre nursing, noticed that patients, who did not receive pre-operative information, appeared to be more anxious than those who had done so. According to Perry and Potter (2002:959) and Clancy, McVicar and Baird (2002:219), anxiety can interfere with the effectiveness of anaesthesia, the patients' abilities to participate in their care as well as their stress and pain levels.

\section{Purpose of the research}

The purpose of the study was to explore and describe the perceptions of orthopaedic patients, who had undergone elective surgery, about the preoperative information provided to them.

\section{Objectives of the research}

The study aimed to identify:

- Whether the orthopaedic patients received information prior to their elective surgery

- Which aspects were included/ not included in the pre-operative information education
- How pre-operative information was conveyed to orthopaedic patients

- Who provided pre-operative information to orthopaedic patients.

\section{Research design}

A non-experimental, exploratory, descriptive and quantitative research design was used. This study was nonexperimental because no variables were manipulated. It was descriptive because structured interviews were conducted with 50 orthopaedic patients requesting information about their perceptions concerning the pre-operative information they had received. The responses to questions on the completed structured interview schedules were coded and quantified. Responses to open-ended questions were grouped and quantified.

\section{Research population}

The population for this study included all orthopaedic patients admitted for elective surgery at all the provincial hospitals in the KwaZulu-Natal Province from 10 May till 27 July 2007. The target population comprised all orthopaedic patients admitted for elective surgery over a period of ten weeks ( 10 May till 19 July 2007) at the two participating provincial hospitals in the KwaZulu-Natal Province.

\section{Sample}

It could not be predetermined whether or not specific orthopaedic patients would be willing to be interviewed about their perceptions relating to preoperative information and no census existed of persons scheduled for elective orthopaedic surgery at these hospitals. Therefore a random sample could not be selected.

All patients, aged 18 or older, who were admitted to the orthopaedic wards at the two participating hospitals for elective surgery, from 10 May till 27 July 2007 , were requested to participate in the study. Orthopaedic patients were selected because post-operatively they are usually well enough to be interviewed and because they require preoperative information. To be eligible for this study, patients had to:

- bel 8 years of age or older 
remain in the hospital after surgery for at least 48 hours

- $\quad$ be medically fit to answer questions

- be able to speak English

- be admitted for elective ortho paedic procedures

- $\quad$ agree to participate voluntarily in the study

\section{Data collection}

Individual face-to-face structured interviews were conducted to collect the data from 50 orthopaedic patients. Data gathering took ten weeks to complete, complicated by the public servants' strike which took place 1-27 June 2007. Most patients who were booked for elective orthopaedic surgery were either transferred to other hospitals or their procedures were cancelled during this period, making them inaccessible for interviews at the two participating hosptials. Data collection took place from 10 May 2007 till 27 July 2007. On average, five patients were interviewed per week over a period of ten weeks.

\section{Research instrument}

The interview schedules consisted of a combination of open and closed ended questions designed to assess how patients perceived their pre-operative information. The interview schedule comprised the following sections:

Section A collected demographic data from orthopaedic patients, such as gender, age, marital status, educational qualifications and the number of admissions for elective orthopaedic surgery.

Section B consisted of questions about the pre-operative information orthopaedic patients had received.

Section $\mathrm{C}$ consisted of questions relating to the persons who provided information pre-operatively to orthopaedic patients.

Section D's questions attempted to determine whether the information provided to orthopaedic patients helped them with their post-operative adaptations, expectations and/or recuperation processes.

\section{Validity and reliability}

The instrument was constructed so that it could measure the attributes to be studied, namely the perceptions of orthopaedic patients regarding pre-operative information received prior to their elective surgical procedures, amounting to face validity. Two nurse educators teaching orthopaedic and operating theatre nursing science, who did not participate in the actual study, as well as two nurse researchers, reviewed the interview schedule items to ensure that the questions were relevant, unambiguous and clear, accepting the interview schedule's construct validity.

The content of the interview schedule was derived from literature on pre-operative information. Further information was gathered from discussions with operating room nurses, orthopaedic ward nurses and nurse educators, amounting to content validity.

Reliability was ensured by applying the test-retest method and internal consistency. The interview schedule was pretested by interviewing four orthopaedic patients admitted to the two participating hospitals prior to 1 May 2007, and who could not participate in the actual study. The results of the pretests were compared with those of the actual research. No major discrepancies were observed, amounting to test-retest reliability of the instrument.

\section{Ethical considerations}

The respondents were fully informed about the process and the purpose of the study. Respondents were not coerced to participate in this study and they had the right to withdraw from the study at any stage without incurring any negative consequences whatsoever. An opportunity was provided for each respondent to ask questions and to clarify misunderstandings.

During the data collection process, the respondents' names were not recorded on the interview schedules, ensuring anonymity. The research report only portrays figures, statistics and discussions but no names. All completed interview schedules were secured under lock and key. No respondent was paid.

Permission to conduct the study was obtained from the Kwazulu-Natal Provincial Department of Health, the chief executive officers of the two participating hospitals, and the from the Re- search and Ethics Committee of the Department of Health Studies, University of South Africa. Signed consent was obtained from every respondent.

\section{Method of data analysis}

The quantitative data collected was analysed using the Statistical Package for Social Sciences (SPSS) version 13. A statistician identified and calculated appropriate statistics.

\section{Demographic data}

Of the 50 respondents, $34(68.0 \%)$ were males and $16(32.0 \%)$ were females, only $10(20.0 \%)$ respondents were married and $25(50.0 \%)$ were between 18 and 38 years of age. Most of these patients were actively involved in contact sports such as soccer, while some were involved in car accidents. Only $6(12.0 \%)$ respondents were between 49 and 68 years of age, of whom most had sustained falls in their homes.

No respondent had a tertiary education qualification and $25(50.0 \%)$ had completed grades 3-10. More than half $(64.0 \% ; n=32)$ of the respondents had no previous experience with orthopaedic surgery while $18(36.0 \%)$ respondents had been admitted for surgery for the second or third time. Of the respondents $35(70.0 \%)$ had sustained injuries to the lower limbs and 15 $(30.0 \%)$ to the upper limbs. The orthopaedic procedures performed on the respondents included: reduction and internal fixation of the radius/ulna $(30.0 \% ; n=15)$, open reduction and internal fixation of the tibial plateau $(24.0 \% ; n=12)$ and pin and plate fixations of the femur $(18.0 \% ; n=9)$.

\section{Pre-operative information received by orthopaedic patients}

Of the respondents, $39(78.0 \%)$ reported pre-operative information to be useful for their preparation for surgery but only $17(34.0 \%)$ considered this to be the case for their post operative recovery. Similar findings were reported by other researchers (Elkin, Perry \& Potter, 2004:496-497; Lewis et al., 2004:369; Shuldham, Fleming \& Goodman, 2002:666).

\section{Pre-operative information provided the night before surgery}

All respondents $(100 \% ; n=50)$ were re- 
Table 1 Types of pre-operative information provided the night before surgery $(n=50)$

\begin{tabular}{|l|l|l|l|}
\hline INFORMATION PROVIDED & YES & NO & $\%$ (YES) \\
\hline Shower & 50 & 0 & 100 \\
\hline No eating/drinking & 49 & 1 & 98.0 \\
\hline Remove jewelry & 40 & 10 & 80.0 \\
\hline Remove finger and toenail polish & 20 & 30 & 40.0 \\
\hline Shave operative site & 8 & 42 & 16.0 \\
\hline Shampoo hair & 2 & 48 & 4.0 \\
\hline Light supper & 1 & 49 & 2.0 \\
\hline
\end{tabular}

quested to shower and almost all $(98.0 \% ; n=49)$ were informed that they should not eat or drink anything prior to the operation. The following information provided the night before surgery appeared to be lacking as only 20 $(40.0 \%)$ respondents were asked to remove finger and toenail polish, 8 $(16.0 \%)$ were asked to shave the operation site, $2(4.0 \%)$ were asked to shampoo their hair and only $1(2.0 \%)$ respondent was asked to have a light supper. Although all these aspects should be attended to during the preoperative stage, specific pre-operative information and/or preparation could be influenced by the type of surgery and by the surgeon's preferences. For example, some orthopaedic surgeons might prefer their patients not to be shaved pre-operatively because any skin cuts could increase the risk of post-operative infections with potentially hazardous risks for the patients.

\section{Pre-operative information provided the morning of the surgery}

Information provided on the morning of surgery lacked information on shaving of the operative site $(16.0 \% ; n=8)$, removing make-up $(2.0 \% ; n=1)$ and premedication $(2.0 \% ; n=1)$. No respondent was reportedly instructed to empty his/her bladder prior to receiving premedication. However, patients scheduled for major orthopaedic surgery usually have urinary catheters inserted prior to these operations, explaining why this pre-operative instruction might have been irrelevant - at least to some respondents.

Of the respondents $35(70.0 \%)$ were not made aware of the operating room en- to watch the video presentation repeatedly to improve their understanding. $\mathrm{X}$-rays enabled the respondents to see where the actual fractures were and how the operations were going to be performed. Most of the respondents had televisions $(785 ; n=39)$, DVD players $(66.0 \% ; n=33)$ and video machines $(52.0 \% ; n=26)$ at home, making these methods of providing pre-operative information feasible. Kulik (1999:3) and Pager (2005:6) reported that respondents who viewed videotapes of their surgical procedures spent less time in the hospital post-operatively and experienced lower stress levels than those who had not done so.

vironment, implying that only 15 $(30.0 \%)$ respondents received information about the operating room environment.

\section{Patients' preferences for receiving pre-operative information}

All $100 \%(n=50)$ of the respondents received pre-operative information orally whereas $88.0 \%(n=44)$ indicated that $\mathrm{X}$-rays were used during such information sessions.

The respondents' preferences for preoperative information included oral communication $(50.0 \% ; n=25)$, videotapes $(30.0 \% ; n=15), X$-rays $(10.0 \% ; n=5)$ and pamphlets/books $(2.0 \% ; n=1)$. Oral presentations were reportedly simple and easy to understand, and presented possibilities to ask questions. Video recordings (tapes and/or compact discs) were interesting to watch, and afforded opportunities

\section{Patients' satisfaction levels with the pre-operative information received}

Reportedly pre-operative information decreased the anxiety levels about their impending surgery of $39(78.0 \%)$ respondents. As many as $33(66.0 \%)$ respondents were satisfied with the preoperative information they had received.

Persons who provided pre-operative information to orthopaedic patients The respondents reported that the surgeon $(82.0 \% ; n=41)$, ward nurse $(44.0 \%$; $\mathrm{n}=22)$, anaesthetist $(14.0 \% ; \mathrm{n}=7)$ and the operating theatre nurse $(12.0 \%$; $\mathrm{n}=6$ ) were the people who provided preoperative information. No respondent mentioned the physiotherapist. However, in response to a later question 27 $(54.0 \%)$ of the respondents indicated that the physiotherapists did teach

\section{Table 2 Types of pre-operative information provided on the morning of surgery $(n=50)$}

\begin{tabular}{|l|l|l|l|}
\hline INFORMATION PROVIDED & YES & NO & \%(YES) \\
\hline No eating/drinking & 50 & 0 & 100 \\
\hline Shower & 50 & 0 & 100 \\
\hline Remove jewelry & 50 & 0 & 100 \\
\hline Remove underwear & 50 & 0 & 100 \\
\hline Wear theatre clothes & 50 & 0 & 100 \\
\hline Shave operative site & 8 & 42 & 16.0 \\
\hline Remove make-up & 1 & 49 & 2.0 \\
\hline Pre-medication & 1 & 49 & 2.0 \\
\hline Empty bladder & 0 & 50 & 0 \\
\hline
\end{tabular}


them about post-operative exercises. Reportedly demonstrations of active and passive exercises were done but respondents were not shown deep breathing nor coughing exercises. Preoperative information should be provided by members of the multidisciplinary health team, including the surgeon, anaesthetist, nurse and physiotherapist so that the patient can foster realistic post operative expectations and co-operate in his/her recuperation (Fielden, Scott \& Horne, 2003:429-431; Prouty et al., 2006:257).

Although most respondents understood the information, $16(32.0 \%)$ found it difficult to understand medical terms and $27(54.0 \%)$ would have appreciated more opportunities to ask further questions.

Only $58.0 \%(n=29)$ of the respondents received information on pain medication. Reportedly respondents did not receive pre-operative information on general aspects of post-operative care, early ambulation and nutrition.

\section{Conclusions}

Most young orthopaedic respondents required surgery after accidents and most older respondents after sustaining falls in their homes. Although all respondents had reportedly received some pre-operative information, some aspects were not addressed.

Pre-operative information provided on the morning of the surgery did not address the removal of nail polish and make-up, did not familiarise the majority of the respondents with the operating theatre environment and did not request any respondent to empty his/ her bladder prior to being given premedication.

All respondents received pre-operative information orally and most were shown their $\mathrm{x}$-rays during these oral sessions. Prostheses and anatomic models were not used. Many respondents would have appreciated more opportunities for asking questions, and for clarifying medical terms. Although pre-operative information had been provided the reasons for procedures and expected post-operative actions were not always supplied. Only approximately half of the respondents had been informed about post-operative pain medication. Post-operative nutrition, early ambulation, deep breathing and coughing exercises were not addressed.

\section{Recommendations}

Based on the conclusions of this study, recommendations will be provided for enhancing the pre-operative information provided to orthopaedic patients and for conducting future research.

\section{Recommendation for improving the quality of pre-operative information provided to orthopaedic patients}

In addition to the pre-operative information routinely provided to orthopaedic patients, the following recommendations could enhance the quality thereof:

- Information provided on the evening preceding the operation should include shaving of the operation site (depending on the specific surgeon's preferences), washing of hair and removal of nail polish and makeup.

- The patients should also be familiarised with the operation procedures such as going to the waiting area, operating theatre, recovery room and ward/intensive care unit. Patients without urinary catheters must be requested to empty their bladders prior to the administration of pre-medication.

- Pre-operative information should include reasons for procedures and post-operative actions such as early ambulation, post operative nutrition, deep breathing and coughing exercises.

- Pre-operative information sessions should encourage patients to ask questions and to clarify medical terminology.

- $\quad X$-rays should be used during pre-operative information sessions.

- Audiovisual aids and equipment such as prostheses, anatomical models of bones and videotapes/compact discs on orthopaedic procedures, should be provided to supplement pre-operative teaching, whenever possible.

- Written instructions, given to the patients in the form of pamphlets or photocopies, explaining procedures and equipment as well as post-operative expectations enable patients to revise these instructions.

- $\quad$ The physiotherapist should supply a written programme of exercises and provide demonstrations of these exercises, as well as the use of crutches or other orthopaedic aids, pre-operatively. Deep breathing and coughing exercises must be included. The physiotherapist's written instructions should also include guidelines for preventing falls in homes.

- $\quad$ Patients should be educated about expected lifestyle changes after surgery.

\section{Recommendations for future studies}

The recommendations listed in the preceding section should be included in the pre-operative checklists of the hospitals concerned. Once these changes have been implemented the study should be repeated to determine whether or not the pre-operative information provided to orthopaedic patients have actually improved. A periodic audit check should be done of the pre-operative information recorded on a specific patient's file correlated with the patient's report. This could help to ensure that orthopaedic patients receive adequate pre-operative information.

The study should be repeated but should include orthopaedic patients who cannot communicate in English.

Qualitative semi-structured interviews should be conducted with orthopaedic patients to learn about their actual experiences concerning pre-operative information.

Orthopaedic ward nurses and operating theatre nurses should be questioned about the pre-operative information provided by them. Based on this information a feasible plan for providing pre-operative information should be designed, implemented, evaluated and improved on an ongoing basis. 
A multidisciplinary study should be conducted to specify the expected preoperative information to be provided by the surgeon, anaesthetist, ward nurse, operating theatre nurse (if any) and physiotherapist. By avoiding duplication, much time could be saved.

Depending on the research results the surgeon could provide information about the actual surgical procedures; the anaesthetist about pre- and postoperative medication; the ward nurse about the expected pre-operative procedures, the actual process through the operating theatre and immediate postoperative expectations; while the physiotherapist could explain the necessity of deep breathing and coughing exercises as well as the use of any orthopaedic aids and the anticipated post-operative exercises.

\section{Limitations of the study}

- The period of data collection was extended to ten weeks due to the public servants' strike during the data collection phase. Most patients who were booked for elective orthopaedic surgery were either transferred to other hospitals or their operations were cancelled. Possible influences of this strike on patients' responses could not be determined.

- Only orthopaedic patients undergoing elective surgery participated in the study.

- $\quad$ Respondents who could not communicate in English were excluded from the study. These persons might have had similar or different views about their pre-operative information compared to those who could speak English and who participated in this study.

- $\quad$ Only two provincial hospitals participated in this study.

- Only structured interview schedules were used to obtain data from the patients. More indepth and different data might have been obtained by conducting in-depth individual qualitative interviews.

\section{Concluding remarks}

More effective pre-operative information could help orthopaedic patients to participate more effectively in their post-operative care and in their long term rehabilitation. Surgeons, anaesthetists, nurses and physiotherapists should collaborate to avoid duplication but to ensure that each patient receives all the required information - preferably verbally and in written format.

\section{References}

ALTIZER, L 2004: Patient education for total hip or knee replacement. Orthopaedic Nursing. 23(4):283-288.

CLANCY,J; MCVICAR,AJ \& BAIRD, N 2002: Perioperative practice: fundamentals of homeostasis. New York: Routledge.

ELKIN, MK; PERRY,AG \& POTTER, PA 2004: Nursing interventions and clinical skills. $3^{\text {rd }}$ edition. St Louis: CV Mosby.

FIELDEN, JM; SCOTT, S \& HORNE, JG 2003: An investigation of patient satsifaction following discharge after total hip replacement surgery. Orthopaedic Nursing. 22(6):429-436.

FITZPATRICK, E \& HYDE, A 2005: What characterizes the "usual" preoperative education in clinical contexts? Nursing and Health Sciences, 7(4):251-258.

IGNATAVICIUS, DD; WORKMAN, ML \& MISHLER, MA 1999: Medicalsurgical nursing across the health care continuum. $3^{\text {rd }}$ edition. Philadelphia: WB Saunders.

JOHANSSON, K; NUUTILA, L, VIRTANEN, H \& SALANTERA, S. 2005: Pre-operative education for orthopaedic patients: systematic review. Journal of Advanced Nursing. 50(2):212-223.

KULIK, JA 1999: Cutting the stress of surgery. Facts of Life. 4(1):1-7.

LEE, DS \& CHIEN, WT 2002: Preopertive teaching in an acute care ward in Hong Kong: a case study. Contemporary Nurse: a Journal for the Australian Nursing Profession. 13(2-3):1-9.

LEWIS, SM; HEITKEMBER, MM, DIRKSEN, SR 2004: Medical-surgical nursing: assessment and management of clinical problems. $6^{\text {th }}$ edition. St Louis: CV Mosby.

PAGER, CK 2005: Randomized controlled trial of pre-operative information to improve satisfaction with cataract surgery. British Journal of Ophthalmology $89(1): 10-13$.

PELLINI,T; TLUCZEK,A; COLLINS, M; TRIMBORN, S; NORWICH, ENGELKE, ZK \& BROAD, J 1998: Increasing self-efficiency through empowerment: pre-operative education for orthopaedic patients. Orthopaedic Nursing. 17(4):48-59.

PERRY, AG \& POTTER, PA 2002: Clinical nursing skills \& techniques.

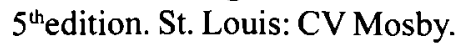

PHILLIPS, NF 2004: Berry and Kohn's operating room technique. $10^{\mathrm{TH}}$ edition. St.Louis: CV Mosby.

PROUTY,A; COOPER, M; THOMAS, P; CHRISTENSEN, J; STRONG, C; BOWIE, L \& OERMANN, MH 2006: Multidisciplinary patient education for total joint replacement surgery patients. Journal of Orthopaedic Nursing. 25(4):257-261.

SMELTZER, SC, \& BARE, BG 2004: Brunner and Suddarth's textbook of medical-surgical nursing. $10^{\mathrm{TH}}$ edition. Philadelphia: JB Lippincott.

SHULDHAM, CM; FLEMING, $S$ \& GOODMAN, H 2002: The impact of pre-operative education on recovery following coronary artery bypass surgery: a randomized controlled clinical trial. European Heart Journal 23(8):666674.

SPALDING, NJ 2004: Pre-operative education: empowering patients with confidence. International Journal of Therapv \& Rehabilitation. 11(4):147152.

WALKER, JA 2007: What is the effect of pre-operative information on patient satisfaction? British Journal of Nursing. 16(1):27-31.

WHYTE, RI \& GRANT, PD 2005: Preoperative patient education in thoracic surgery. Thoracic Surgery Clinics. 15(2):195-201. 